\title{
FONTE E DISTRIBUIÇÃO DE HIDROCARBONETOS DO PETRÓLEO NOS SEDIMENTOS DA BAÍA DE TODOS OS SANTOS, BAHIA
}

\author{
CELINO, J.J. ${ }^{1 *}$; VEIGA, I.G. ${ }^{2}$; TRIGÜIS, J.A. ${ }^{2}$ \& QUEIROZ, A.F.S. ${ }^{1}$ \\ ${ }^{1}$ Núcleo de Estudos Ambientais, Instituto de Geociências \\ Universidade Federal da Bahia (UFBA) \\ Rua Barão de Geremoabo, s/n, Sala 304 A1, Federação, CEP 40170-290 \\ Salvador, Bahia, Brasil \\ E-mail: queiroz@ufba.br, joil@ufba.br * (Autor para contato) \\ ${ }^{2}$ Centro de Ciência e Tecnologia, Universidade Estadual do Norte Fluminense \\ Macaé, Rio de Janeiro, Brasil. \\ E-mail: triguis@lenep.uenf.br
}

\begin{abstract}
Celino, J.J.; Veiga, I.G.; Trigüis, J.A. \& Queiroz, A.F.S. 2008. Source and distribution of petroleum hydrocarbons in sediments from Todos os Santos Bay, Bahia. Braz. J. Aquat. Sci. Technol. 12(1):31-38. ISSN 1808-7035. Todos os Santos Bay, located in Recôncavo Bahiano, is the largest navigable bay of Brazil. Its seafront has about 450 $\mathrm{km}$ and is recovered by extensive mangroves, which have developed over an humid substract rich in argillaceous minerals and organic matter. Coastal marine ecosystems, specially the semi-enclosed systems like bays, are under unrelenting stress caused by urban and industrial development. Mangroves are classified as being the most sensitive coastal environment and can be seriously affected by oil. Petroleum causes alteration on the mangrove that are common to several spills studies. This study was undertaken in the north area of Todos os Santos Bay to evaluate the possibility of chronic environmental impact induced by 50 years of exposure to the local petroleum industry. Pollution was investigated by determination of aliphatic hydrocarbon concentrations and biomarker compounds, for organic matter source identification. Results revealed that the main sources of hydrocarbons are from higher forests. Others sources of anthropogenic hydrocarbons are domestic and industrial effluents, shipping operations, offshore petroleum production, and transportation.
\end{abstract}

Keywords: Biomarker; Aliphatic Hydrocarbon; Sediments; Mangrove; Marine Ecosystems

\section{INTRODUÇÃO}

A Baía de Todos os Santos (BTS), localizada no Recôncavo Baiano, é a maior baía navegável do Brasil. Com aproximadamente $450 \mathrm{Km}$ de extensão, sua orla é recoberta por extenso manguezal que assistiram à implantação das primeiras unidades de exploração, produção e refino de petróleo em território brasileiro. Suas características são claramente marinhas, pois o volume de água doce, oriunda dos diversos cursos fluviais que nela deságuam, são duas ordens de grandeza inferior ao aporte de água salgada que entra pela abertura da baía (Leão \& Dominguez, 2000). Tratase de uma baía fortemente influenciada pelas massas oceânicas, com salinidade variando entre 28 e $36 \%$. Alguns fatores morfológicos (valores médios) contribuem para o baixo tempo de residência da água no seu interior: profundidade de 6,9 metros, volume de 6,39x $10^{9} \mathrm{~m}^{3}$ e correntes com velocidade de $41,0 \mathrm{~cm} \cdot \mathrm{s}^{-1}$. (Orge et al., 2000).

Com o objetivo de analisar a proveniência dos hidrocarbonetos estocados, a série homóloga dos alcanos normais foi identificada nas amostras dos sedimentos superficiais de zonas de manguezal da região norte da Baía de Todos os Santos - BTS (Fig. 1) entre os compostos $n \mathrm{C} 14-n \mathrm{C} 32$. Os alcanos normais são compostos facilmente intemperizados no ambiente marinho e sua presença em proporções consideravelmente altas podem ser indicativas de aporte recente de hidrocarbonetos fósseis (Woohead et al., 1999). Assim sendo, com o objetivo de verificar esta hipótese, a contribuição relativa dos alcanos normais na composição dos HTP (hidrocarbonetos totais de petróleo) e HRP (hidrocarbonetos resolvidos de petróleo) foi determinada (Celino et al., 2005).

Os índices preferenciais de carbono (IPC) foram calculados para diversas faixas de compostos, segundo as equações descritas em Le Dréau et al. (1997). A determinação do IPC é uma medida comparativa da contribuição de $n$-alcanos derivados de síntese biogênica relacionada a vegetais superiores (IPC $>1$ ) e hidrocarbonetos antropogênicos (IPC $<1$ ), indicativo de poluição ambiental por petróleo (Aboul-Kassim \& Simoneit, 1995). Adicionalmente foram calculadas 
razões entre as concentrações dos compostos $n \mathrm{C} 29 \mathrm{e}$ $n \mathrm{C} 17$.

\section{MÉTODOS EXPERIMENTAIS}

\section{Amostragem}

Baseando-se em estudos anteriores (Tavares, 1997; Peso-Aguiar et al., 2000; Orge et al., 2000) foi feito inicialmente um levantamento geoquímico em oito estações (Fig. 1). Após a aquisição destes dados analíticos, o número de estações de coleta foi ampliado. Para fins de comparação, foi definida uma estação controle, localizada na llha do Pecado (Baía do Iguape, município de Maragojipe), com características fisiográficas, hidrodinâmicas e ecológicas semelhantes às anteriores, contudo sem atividades petrolíferas nas suas proximidades.

As estações de amostragem foram agrupadas em função das atividades da indústria petrolífera (Fig. 1), a saber: Área de Produção (Dom João, llha de Cajaíba, Subaé, município de São Francisco do Conde), Área de Refino (Coqueiro Grande 1 e 2), Área Portuária (Madre Deus, Caípe, e Suape) e Área Controle (Maragojipe).
A amostragem consistiu na coleta sistemática de sedimentos superficiais até $20,0 \mathrm{~cm}$ do substrato do manguezal, em região de inter-maré, em locais selecionados como mostrado na figura 1, nos períodos de novembro de 2001 e setembro de 2002 (Veiga, 2003). Utilizou-se uma colher metálica lavada na água do manguezal recolhendo amostras dos sedimentos superficiais com massa de aproximadamente 500 gramas, acondicionadas em vasilhames de vidro com tampas metálicas e imediatamente resfriadas à temperaturas em torno de $0^{\circ} \mathrm{C}$.

\section{Preparação das Amostras}

Uma massa de $10 \mathrm{~g}$ da amostra de sedimento, sem tratamento prévio, foi fortalecida com uma solução de hidrocarbonetos saturados normais a concentrações definidas e extraída com hexano ultra puro em soxhlet. O extrato orgânico foi então concentrado em um concentrador de célula fechada do tipo Kuderna Danish a um volume de $1 \mathrm{~mL}$. A massa de óleo foi determinada neste concentrado por gravimetria, pesado após evaporação do $1 \mathrm{~mL}$ de extrato e a fração de hidrocarbonetos saturados foi separada deste extrato através do tratamento do mesmo com uma coluna de sílica gel ativada. (Veiga 2003; Celino \& Queiroz, 2006).

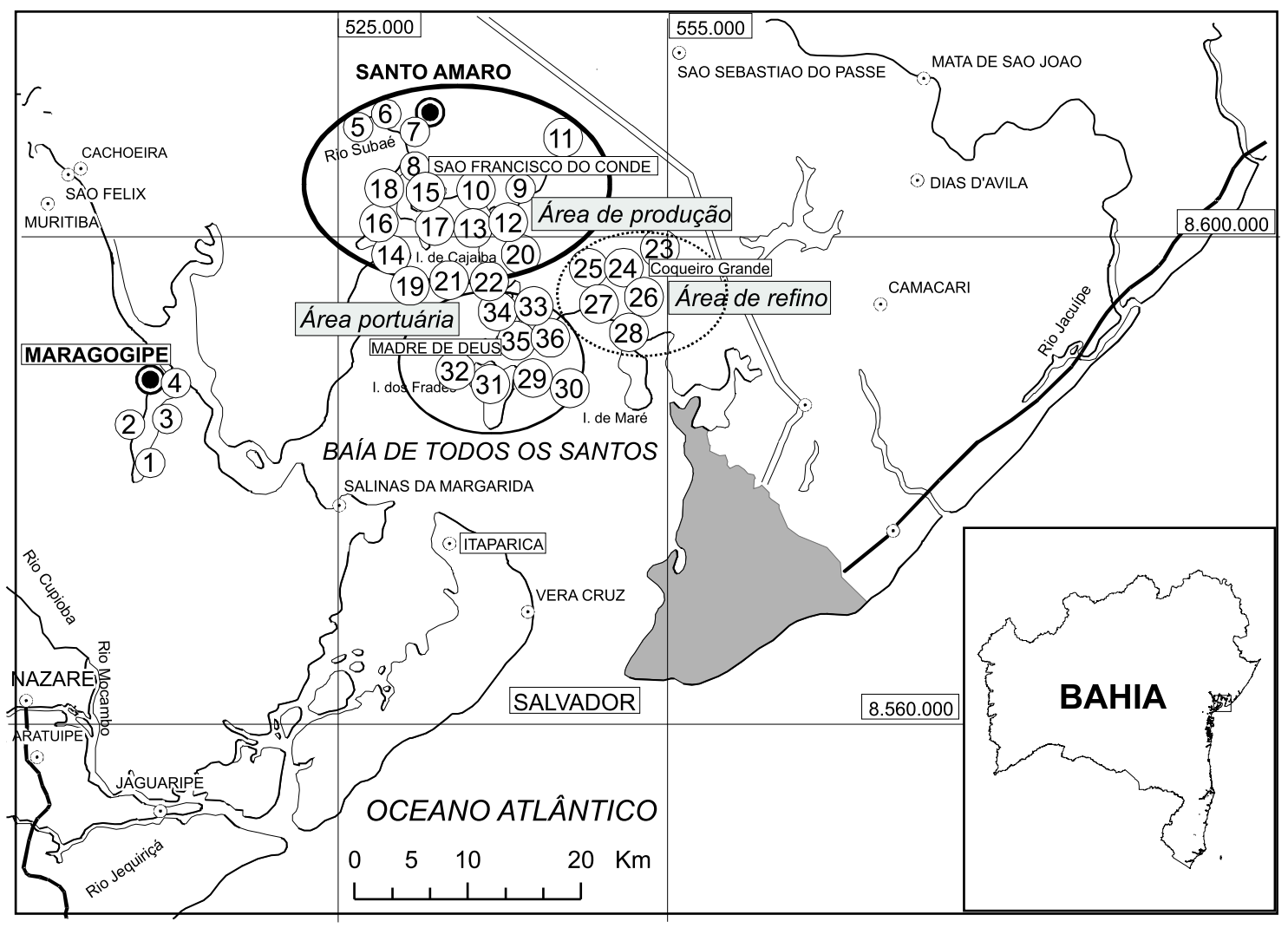

Figura 1 - Mapa de situação e de localização das estações de amostragem dos sedimentos na BTS agrupadas por atividade principal (áreas de produção, refino e portuária). 


\section{MÉTODOS ANALÍTICOS}

A fração de hidrocarbonetos saturados foi separada deste extrato $(1 \mathrm{~mL})$ através do tratamento do mesmo com uma coluna de sílica gel ativada - uma bureta de $25 \mathrm{ml}(1 \mathrm{~cm} \times 45 \mathrm{~cm})$ contendo $3 \mathrm{~g}$ de sílica gel firmemente compactada (72-230 mesh, Sigma) e uma camada de $1-2 \mathrm{~cm}$ de $\mathrm{Na}_{\mathrm{s}} \mathrm{SO}_{4}$ na superfície para absorver a água - e eluição com $20 \mathrm{~mL}$ de hexano ultra puro (Merck). A fração de hidrocarbonetos aromáticos foi separada através da eluição com uma mistura de $60 \mathrm{ml}$ de hexano/diclorometano (1:1 v/v) da mesma coluna, e a fração de NSO (resinas e asfaltenos) foi separada através da eluição do mesmo extrato na coluna com 15 $\mathrm{mL}$ de metanol (Merck). Todas as frações foram determinadas por gravimetria.

O concentrado final foi diretamente injetado, sem divisão de fluxo, em uma coluna de fase estacionária 30m DB-5 instalada em um cromatógrafo a gás HP 6890. A programação de cromatografia gasosa (CG) dos hidrocarbonetos saturados é descrita a seguir: injeção sem divisão de fluxo, com o injetor a $280^{\circ} \mathrm{C}$; temperatura inicial da coluna $50^{\circ} \mathrm{C}$, isoterma por 1 minuto, taxa de aquecimento de $6^{\circ} \mathrm{C} / \mathrm{min}$ até a temperatura final de $310^{\circ} \mathrm{C}$, isoterma de 10 minutos.

$A$ análise quantitativa de biomarcadores saturados foi realizada utilizando-se a técnica de cromatografia gasosa acoplada a espectrometria de massas (CG/EM), utilizando-se a fração de hidrocarbonetos saturados (Tab. 1). Os biomarcadores do tipo terpanos pentacíclicos e esteranos foram analisados através da técnica de monitoramento seletivo de íons, utilizando-se os íons m/z 191 e m/z 217 como íons diagnósticos dos biomarcadores (Tab. 2).

\section{RESULTADOS E DISCUSSÃO}

As concentrações totais de alcanos normais (“ $n C 14-n C 32$ ) variaram entre 1035,84 e 5728,68 ng/g, nas Áreas Portuária e Produção, respectivamente (Tab. 1). Foram identificados picos de concentração elevada na Ilha de Cajaíba $(36.507,27$ ng/g) e Dom João $(9.175,77 \mathrm{ng} / \mathrm{g})$, ambas na área de produção.

A tabela 3 exibe os parâmetros estatísticos básicos (média aritmética, média geométrica, mediana, etc.), para as 36 amostras de toda a área.

Tabela 1 - Concentração dos $n$-alcanos ( $\mathrm{ng} / \mathrm{g}$ ) nos sedimentos superficiais da região norte da BTS.

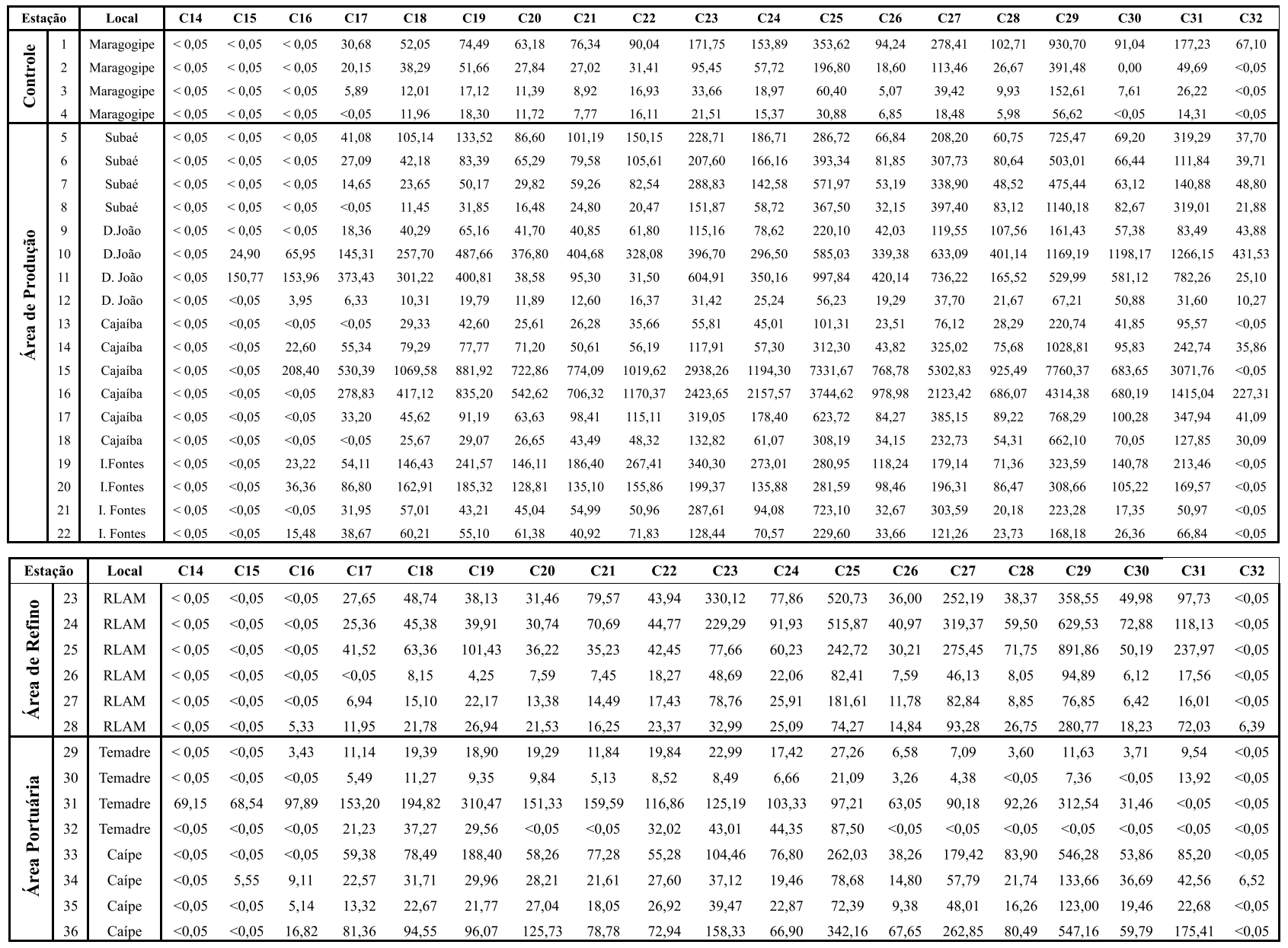


Tabela 2 - Razões diagnósticas com hidrocarbonetos saturados cíclicos para os sedimentos superficiais de zonas de manguezal da região norte da Baía de Todos os Santos.

\begin{tabular}{|c|c|c|c|c|c|c|c|c|c|c|}
\hline \multirow[t]{2}{*}{ RAZÕES } & \multicolumn{5}{|c|}{$\begin{array}{c}\text { ÁREA } \\
\text { DE PRODUÇÃo }\end{array}$} & \multicolumn{2}{|c|}{$\begin{array}{l}\text { ÁREA DE } \\
\text { REFINO }\end{array}$} & \multicolumn{2}{|c|}{$\begin{array}{c}\text { ÁREA } \\
\text { PORTUÁRIA }\end{array}$} & \multirow{2}{*}{$\begin{array}{c}\text { ÁREA } \\
\text { CONTROLE } \\
\text { Maragojipe }\end{array}$} \\
\hline & Subaé & D.João & D.João & Cajaíba & I.Fontes & C.G. & C.G. & Madre Deus & Caípe & \\
\hline Hop/Ster & 8,14 & 29,18 & 11,06 & 22,68 & 9,69 & 11,45 & 26,50 & 8,20 & 8,80 & 6,85 \\
\hline Tri/Hopanos & 1,30 & 0,72 & 0,70 & 0,99 & 1,25 & 1,42 & 0,95 & 1,00 & 0,88 & 1,89 \\
\hline $\mathrm{Ts} /(\mathrm{Ts}+\mathrm{Tm})$ & 0,55 & 0,66 & 0,53 & 0,68 & 0,61 & 0,54 & 0,59 & 0,53 & 0,60 & 0,49 \\
\hline Norneo/H29 & 0,38 & 0,64 & 0,45 & 0,06 & 0,63 & 0,26 & 0,28 & 0,33 & 0,41 & 0,18 \\
\hline $\mathrm{H} 28 / \mathrm{H} 29$ & 0,00 & 0,00 & 0,00 & 0,00 & 0,00 & 0,00 & 0,00 & 0,00 & 0,00 & 0,00 \\
\hline $\mathrm{H} 29 / \mathrm{H} 30$ & 0,53 & 0,40 & 0,40 & 0,43 & 0,43 & 0,45 & 0,51 & 0,61 & 0,58 & 0,76 \\
\hline OL/H30 & 0,00 & 0,00 & 0,00 & 0,00 & 0,00 & 0,00 & 0,00 & 0,00 & 0,00 & 0,00 \\
\hline GAM/H30 & 0,23 & 0,12 & 0,21 & 0,36 & 0,32 & 0,35 & 0,57 & 0,33 & 1,00 & 0,00 \\
\hline $\mathrm{H} 35 / \mathrm{H} 34$ & 0,89 & 0,44 & 0,48 & 0,59 & 0,51 & 0,69 & 0,47 & 0,68 & 0,52 & 0,92 \\
\hline 25nor/Hopano & 0,00 & 0,00 & 0,00 & 0,00 & 0,00 & 0,00 & 0,00 & 0,00 & 0,00 & 0,00 \\
\hline TET24/26Tri & 0,76 & 0,84 & 0,71 & 0,94 & 0,91 & 0,42 & 0,20 & 0,70 & 0,45 & 1,28 \\
\hline $21 / 23 \operatorname{Tri}$ & 1,07 & 1,65 & 1,18 & 1,34 & 1,14 & 1,05 & 1,40 & 0,95 & 1,39 & 0,70 \\
\hline $26 / 25$ tri & 1,18 & 0,93 & 0,94 & 0,59 & 0,36 & 0,82 & 1,38 & 0,74 & 1,15 & 0,43 \\
\hline $20 S /(20 S+20 R)$ & 0,62 & 0,64 & 0,46 & 0,00 & 0,30 & 0,59 & 0,62 & 0,54 & 0,58 & 0,41 \\
\hline$\beta ß /(a a+\beta ß)$ & 0,44 & 0,23 & 0,29 & 0,64 & 0,11 & 0,47 & 0,40 & 0,45 & 0,39 & 0,49 \\
\hline$\%$ de 27 & 44,81 & 30,64 & 39,72 & 33,60 & 41,22 & 41,04 & 28,99 & 33,90 & 31,68 & 45,41 \\
\hline$\%$ de 28 & 23,78 & 24,35 & 24,36 & 23,08 & 22,81 & 23,85 & 29,70 & 24,68 & 26,03 & 23,70 \\
\hline$\%$ de 29 & 31,41 & 45,01 & 35,92 & 43,32 & 35,97 & 35,10 & 41,31 & 41,43 & 42,29 & 30,89 \\
\hline
\end{tabular}

Tabela 3 - Alguns parâmetros estatísticos básicos (Médias aritméticas $=M A$, médias geométricas $=M G$, médias harmônica $=$ $\mathrm{MH}$, medianas $=\mathrm{MDN}$, valor mínimo $=\mathrm{VMN}$, valor máximo $=\mathrm{VMX}$, desvio padrão = DP e Variância) para o conjunto das 36 amostras de sedimento do manguezal da Baía de Todos os Santos - BA.

\begin{tabular}{|c|c|c|c|c|c|c|c|c|}
\hline & MA & MG & MH & MD & VMN & VMX & DP & Variância \\
\hline C15 & 97 & 90 & 65 & 102 & 5,5 & 151 & 23 & 514 \\
\hline C16 & 81 & 55 & 24 & 101 & 3,4 & 208 & 47 & 2204 \\
\hline C17 & 77 & 40 & 23 & 36 & 5,5 & 530 & 109 & 11885 \\
\hline C18 & 103 & 48 & 29 & 44 & 8,2 & 1070 & 189 & 35618 \\
\hline C19 & 135 & 62 & 33 & 51 & 4,3 & 882 & 209 & 43831 \\
\hline C20 & 91 & 45 & 29 & 37 & 7,6 & 723 & 151 & 22699 \\
\hline $\mathbf{C 2 1}$ & 104 & 48 & 26 & 53 & 5,1 & 774 & 173 & 29817 \\
\hline C22 & 125 & 55 & 36 & 47 & 8,5 & 1170 & 249 & 61930 \\
\hline C23 & 295 & 124 & 65 & 127 & 8,5 & 2938 & 604 & 365010 \\
\hline C24 & 180 & 74 & 42 & 69 & 6,7 & 2158 & 394 & 155389 \\
\hline C25 & 574 & 229 & 119 & 271 & 21,1 & 7332 & 1313 & 1724421 \\
\hline C26 & 107 & 40 & 20 & 37 & 3,3 & 979 & 208 & 43405 \\
\hline C27 & 397 & 152 & 53 & 188 & 4,4 & 5303 & 915 & 836882 \\
\hline C28 & 108 & 51 & 26 & 66 & 3,6 & 925 & 187 & 34960 \\
\hline C29 & 728 & 307 & 97 & 341 & 7,4 & 7760 & 1408 & 1982021 \\
\hline C30 & 137 & 65 & 30 & 61 & 0 & 1198 & 249 & 61937 \\
\hline C31 & 284 & 106 & 51 & 101 & 9,5 & 3072 & 572 & 327318 \\
\hline C32 & 89 & 66 & 40 & 101 & 6,4 & 432 & 73 & 5305 \\
\hline
\end{tabular}

A maior parte dos elementos analisados apresenta assimetria positiva. As medianas são mais próximas da média geométrica do que da média aritmética (Tab. 3). Estes dois aspectos evidenciam a tendência à lognormalidade da distribuição.

A identificação dos picos de maior concentração dos alcanos normais é um fator importante na avaliação de procedência da matéria orgânica, uma vez que cada grupo de organismos imprime um registro específico nos cromatogramas da fração dos hidrocarbonetos saturados (Aboul-Kassim \& Simoneit, 1995). De um modo geral, a quantificação de alcanos normais (" $n \mathrm{C} 14$ - $n$ C32) mostrou uma distribuição com predominância de valores máximos de concentração em $n \mathrm{C} 25$ e $n \mathrm{C} 29$ (Fig. 2). Foram também registrados picos máximos em $n \mathrm{C} 23, n \mathrm{C} 27$ e $n \mathrm{C} 31$ em llha das Fontes e Dom João, respectivamente.

Para a Área de Produção, os diagramas (Fig. 3) apresentam uma distribuição de $n$-alcanos variando entre $n \mathrm{C} 15$ a $n \mathrm{C} 32$, com predominância de $n$-alcanos de alto peso molecular ( $>n C 20$ ), e concentrações de $n$-alcanos ímpares maiores que as dos pares consecutivos (Fig. 3). Pode-se observar a existência de uma rampa correspondente aos compostos não resolvidos por cromatografia gasosa (MCNR) com comportamento bimodal, indicativo da presença de compostos de difícil degradação. O diagrama (Fig. 3) exibe uma feição típica de mistura de contribuição matéria orgânica, ilustrada pela depleção acentuada dos compostos entre $n \mathrm{C} 20-n \mathrm{C} 22, n \mathrm{C} 28$ e $n \mathrm{C} 32$.

$\mathrm{Na}$ Área de Refino, foram identificados $n$-alcanos entre $n \mathrm{C} 16-n \mathrm{C} 32$ sendo que, em alguns locais, o isoprenóide pristano está ausente (Fig. 4). Os compostos leves $(<n C 20)$ estão fortemente depletados, nada se podendo concluir com relação à sua origem. Os picos de máximas concentrações correspondem aos compostos $n \mathrm{C} 25$ e $n \mathrm{C} 29$, com compostos ímpares predominando sobre os pares, típico dos vegetais superiores (manguezal) - (Lê Dréau et al., 1997). 
Normalmente, observa-se a ausência de rampa correspondente à MCNR.

Dentre todas as áreas estudadas, aquela que apresenta a distribuição de $n$-alcanos mais irregular é a área Portuária (Tab. 1 e Fig. 5). Os alcanos normais foram identificados entre $n \mathrm{C} 14-n \mathrm{C} 32$, com ausência de alguns compostos no intervalo considerado. De modo geral, predominam os compostos leves sobre os pesados, o que é um possível indicativo de aporte recente de hidrocarboneto. Os picos de concentração máxima referem-se aos compostos $n \mathrm{C} 19, n \mathrm{C} 25$ e $n \mathrm{C} 29$ (Fig. 5).

Na Área Controle (Maragojipe), os alcanos normais foram identificados entre os compostos $n \mathrm{C} 17$ $-n \mathrm{C} 32$, com picos em $n \mathrm{C} 29$ (Fig. 6). As frações leves foram severamente intemperizadas e ocorrem subordinadamente às pesadas.

Visando detectar as associações de variáveis executou-se a análise de agrupamento hierárquico para 19 variáveis, utilizando o método aglomerativo e a regra de agrupamento de Ward. Para a medida da distância de amalgamação foi usado 1-Pearson-r. O método aglomerativo começa considerando cada observação como um agrupamento separado e prossegue combinando-as até que todas as observações pertençam a um agrupamento.

$O$ resultado da análise de agrupamento hierárquico está representado na figura 7. Para a distância de amalgamação de 0.6 observam-se 3 grupos de variáveis:
1) $\mathrm{C} 17$ - $\mathrm{C} 32$ - $\mathrm{C} 16-\mathrm{C} 15-\mathrm{C} 14$
2) $\mathrm{C} 22-\mathrm{C} 26-\mathrm{C} 19-\mathrm{C} 18$
3) $\mathrm{C} 28$ - $\mathrm{C} 21-\mathrm{C} 20$

Segundo Celino et al. (2005), os alcanos normais representam entre 1,17\% (Área Portuária) e 12,20\% (Área Controle) dos HTP, com valores extremos detectados em Dom João $(0,35 \%)$ e Maragojipe $(35,4 \%)$. No que diz respeito à composição dos HRP, a fração dos alcanos normais é mais representativa, variando entre $18,77 \%$ (portuária) e 27,63\% (controle). Os maiores percentuais de alcanos normais relativos aos HRP ocorrem em Dom João, Ilha de Cajaíba, Coqueiro Grande e Madre Deus, localidades mais próximas das Áreas de Produção, Refino e Portuária e, conseqüentemente mais expostas aos derrames acidentais. A existência de valores elevados de

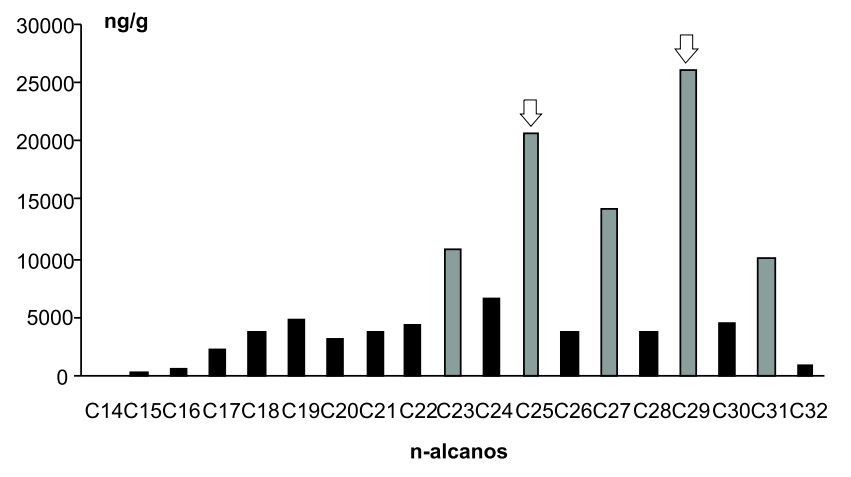

Figura 2 - Distribuição total de hidrocarbonetos saturados da área estudada.

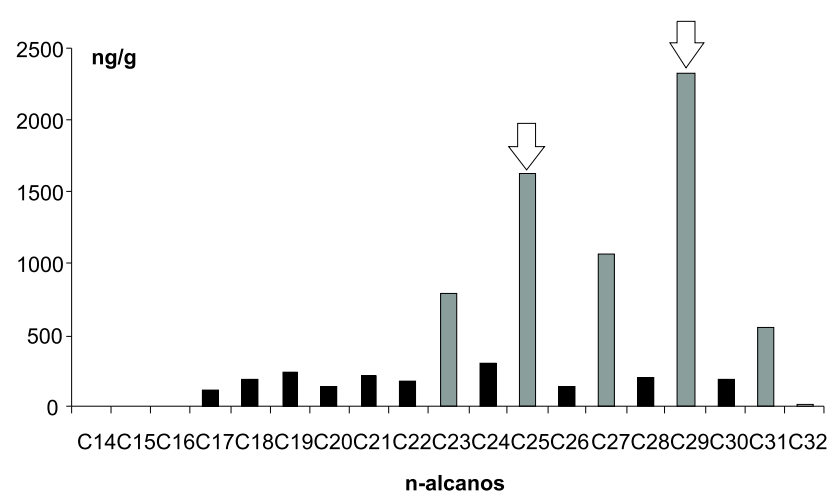

Figura 4 - Distribuição de hidrocarbonetos saturados da área de Refino.

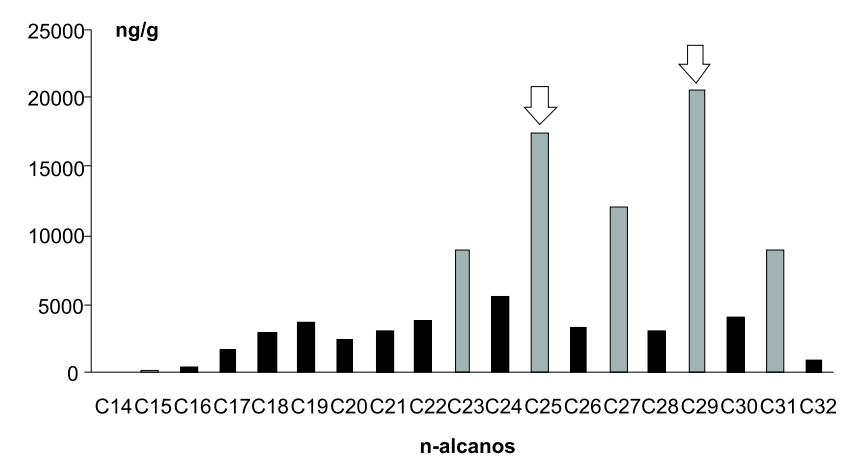

Figura 3 - Distribuição de hidrocarbonetos saturados da área de Produção.

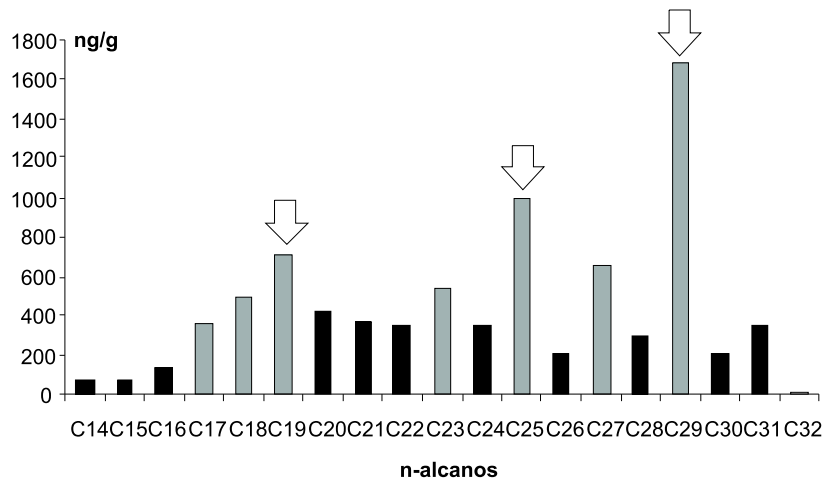

Figura 5 - Distribuição de hidrocarbonetos saturados da área Portuária. 
concentração de alcanos normais na composição dos HRP, em Subaé, pode ser indicativa da presença de hidrocarbonetos antropogênicos recentes, decorrentes de lançamentos de esgotos domésticos e/ou industriais ao longo do curso do rio Subaé.

O índice IPC geral ou IPC ${ }_{14-36}$ foi calculado considerando-se todos os compostos da série homóloga dos alcanos normais ( $n \mathrm{C} 14-n \mathrm{C} 36)$, identificados nos diagramas das amostras ambientais e apresentou valores muito superiores à unidade em todas as áreas, variando entre 4,31 (Área Portuária) e 10,90 (Área Controle) (Tab. 4). Esses resultados indicam uma predominância de hidrocarbonetos derivados de matéria orgânica recente nos sedimentos. Maiores detalhes quanto à procedência da matéria orgânica estocada nos sedimentos podem ser obtidos por meio dos demais índices IPC (Tab. 4), calculados para os hidrocarbonetos saturados normais compreendidos entre os intervalos $n \mathrm{C} 16-n \mathrm{C} 20$ (IPC $\left.{ }_{16-20}\right), n \mathrm{C} 22-n \mathrm{C} 26$ (IPC ${ }_{22-26}$ ) e $n$ C26 $-n$ C36 (IPC ${ }_{26-36}$ ). Em todas as localidades verificouse a seguinte relação: IPC ${ }_{16-20}<I_{22-26}<I_{26-36}$.

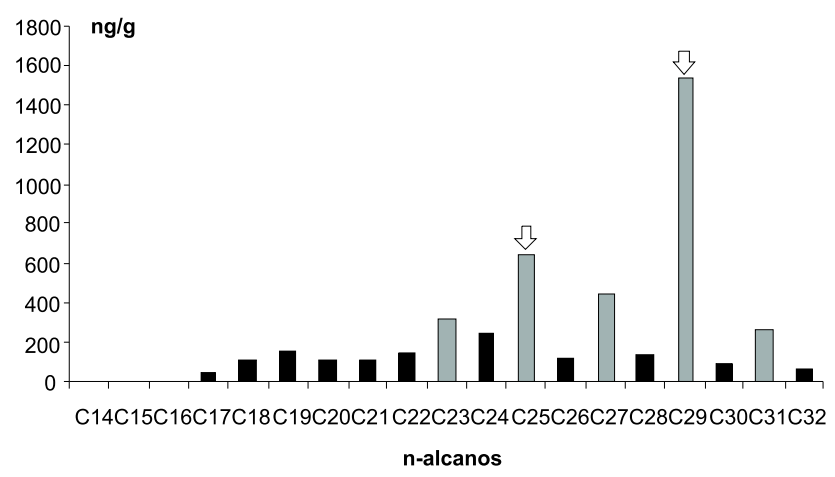

Figura 6 - Distribuição de hidrocarbonetos saturados da área de Controle (Maragojipe).
Valores de IPC ${ }_{26-36}$ superiores a 4, indicando uma possível origem da matéria orgânica sedimentar a partir da vegetação superior continental, foram predominantemente identificados em todas as áreas.

Quanto ao IPC ${ }_{16-20}$, verificou-se que em todas as estações os valores médios calculados são aproximadamente iguais a 1 , variando de 1,19 $\pm 0,48$ (Área Refino) a 1,44 $\pm 0,41$ (Área Produção) (Tab. 4). Esses valores sugerem uma contribuição de hidrocarbonetos de origem antrogopênica na faixa do peso molecular em pauta, que podem estar relacionados com derivados de petróleo - diesel ou óleo lubrificante. No entanto, por se tratar de compostos facilmente intemperizáveis no meio ambiente marinho tropical, os resultados devem ser vistos com cautela.

A razão entre $n \mathrm{C} 29$ e $n \mathrm{C} 17$ (Tab. 4) tem sido usada para avaliar a predominância de compostos de hidrocarbonetos biogênicos de origem marinha ou continental. Se a razão $n \mathbf{C 2 9} / n \mathbf{C 1 7}>1$ tem-se que a principal fonte de alcanos é relativa ao meio continental, caso contrário, predomina no ambiente o aporte aquático marinho, representado pelo $n C 17$ (Lê Dréau et al., 1997). Em todas as estações, as razões $n \mathbf{C 2 9} / \boldsymbol{n C 1 7}$ determinadas foram muito maiores que a unidade, variando entre 4,44 $\pm 3,77$ e 18,92 \pm 13,38 na área Portuária e Controle, respectivamente, denotando mais uma vez a predominância da contribuição da matéria orgânica continental recente, relacionada provavelmente à vegetação do manguezal, no conteúdo total dos hidrocarbonetos sedimentares. Com relação às razões que envolvem concentrações de $n$-alcanos, deve-se atentar para o fato de que os compostos mais leves ( $\boldsymbol{n C 1 7}$, por exemplo) são mais facilmente intemperizáveis que os hidrocarbonetos de maior peso molecular ( $\boldsymbol{n} \mathbf{C 2 9}$, por exemplo), fato este que afeta as determinações de suas concentrações em amostras

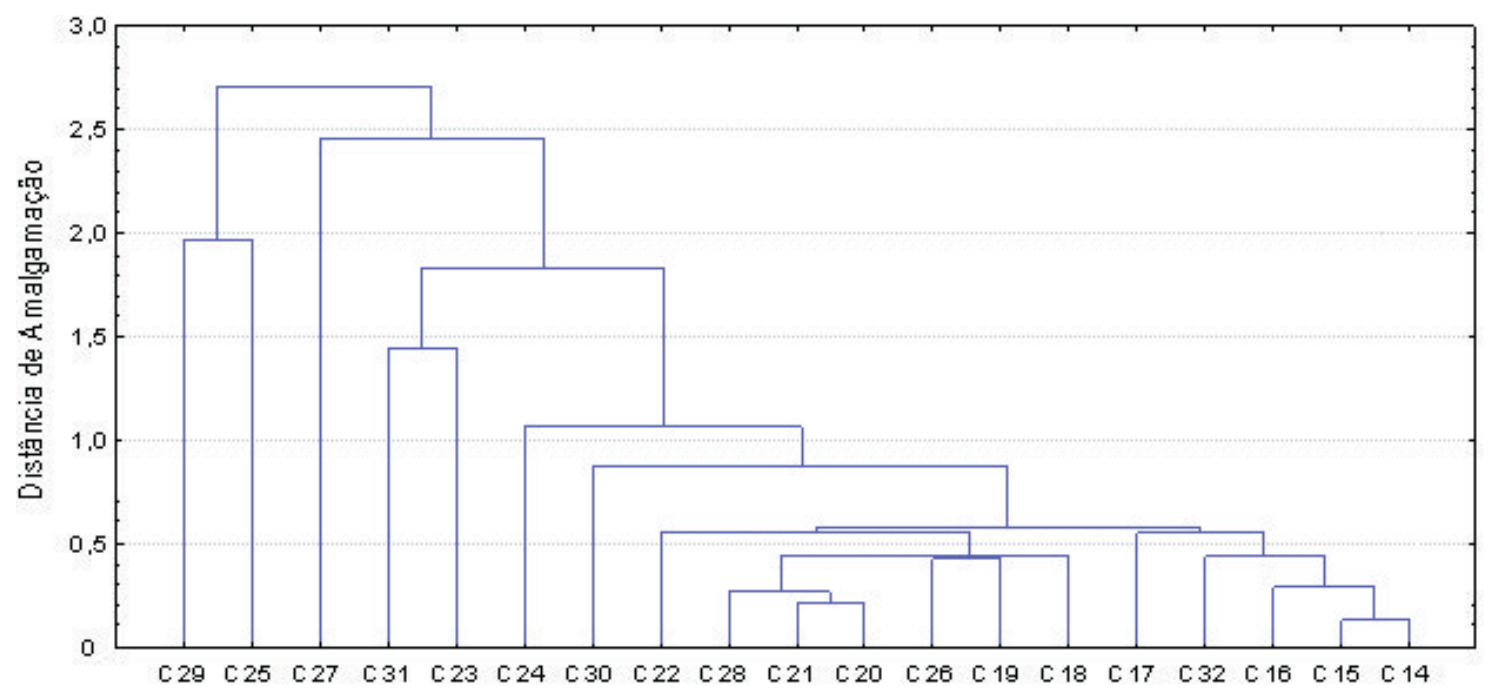

Figura 7 - Diagrama de agrupamento hierárquico calculado para os resultados analíticos de 19 variáveis, em 36 amostras. 
Tabela 4 - Índices IPC e razão $n \mathrm{C} 29$ / $n \mathrm{C} 17$ para diferentes intervalos de alcanos normais em sedimentos superficiais do substrato de zonas de manguezal na região norte da Baía de Todos os Santos (valores médios e desvio padrão).

\begin{tabular}{|c|c|c|c|c|}
\hline PARÂMETROS & $\begin{array}{c}\text { ÁREA DE } \\
\text { PRODUÇÃO }\end{array}$ & $\begin{array}{c}\text { ÁREA DE } \\
\text { REFINO }\end{array}$ & $\begin{array}{c}\text { ÁREA } \\
\text { PORTUÁRIA }\end{array}$ & $\begin{array}{c}\text { ÁREA } \\
\text { CONTROLE }\end{array}$ \\
\hline IPC \\
$\mathbf{1 6}_{-20}$ & $1,44 \pm 0,41$ & $1,19 \pm 0,48$ & $1,33 \pm 0,50$ & $1,39 \pm 0,14$ \\
\hline $\mathbf{I P C}_{\mathbf{2 2}-26}$ & $3,22 \pm 1,68$ & $4,81 \pm 1,89$ & $2,51 \pm 0,78$ & $2,74 \pm 0,68$ \\
\hline $\mathbf{I P C}_{\mathbf{2 6}-36}$ & $5,26 \pm 2,93$ & $8,44 \pm 1,35$ & $4,17 \pm 1,33$ & $10,90 \pm 4,05$ \\
\hline $\mathbf{I P C}_{14-36}$ & $6,14 \pm 3,25$ & $9,39 \pm 1,37$ & $4,31 \pm 1,47$ & $7,24 \pm 1,78$ \\
\hline $\mathbf{n C}_{29} / \mathbf{n C}_{\mathbf{1 7}}$ & $10,57 \pm 9,10$ & $15,64 \pm 9,52$ & $4,44 \pm 3,77$ & $18,92 \pm 13,38$ \\
\hline
\end{tabular}

ambientais e, conseqüentemente, o cálculo das razões diagnósticas.

Com exceção de Maragojipe, onde predominam os compostos tetracíclicos terpanos, nas demais estações verifica-se a predominância dos compostos tricíclicos terpanos (Tab. 2). Esse resultado é indicativo da presença de produtos refinados derivados de petróleo nos sedimentos dos manguezais de Maragojipe, estação controle.

Compostos da série dos hopanos entre $\mathrm{C} 27 \mathrm{e}$ C33 com configuração predominante $17 \mathrm{a}(\mathrm{H}), 21 \mathrm{~B}(\mathrm{H})$, caracterizando uma origem petrogênica foi identificada em todas as estações. A série hopanóide com homólogos entre C31 e C35, com epimerização no carbono $22(S+R)$ e a presença de gamacerano subordinadamente, é típica de hidrocarbonetos de origem fóssil (Peters \& Moldowan, 1993) e foram vistas em todas as estações, com padrões de distribuição condizentes com a presença de óleo nos manguezais de Maragojipe, Estação Controle. Quanto aos esteranos, identificados pela razão $\mathrm{m} / \mathrm{z}=217$, não foi observada uma distribuição uniforme entre os compostos, com predominância ora do C29 esterano, ora do $\mathrm{C} 27$, evidenciando uma mistura de óleos com aspectos geoquímicos diferentes (Tab. 2).

Em todas as localidades foram observadas as razões: Oleanano $/ \mathrm{H} 30=0$, hopanos / esteranos $>5$, $\mathrm{H} 29 / \mathrm{H} 30<1$, ausência de 25NorHopano e presença de tricíclicos terpanos, que em conjunto são indicativas de óleo de origem de ambiente lacustre com rocha geradora siliciclástica de idade pré-aptiana e não biodegradado, características semelhantes ao óleo produzido na Bacia do Recôncavo (Tab. 2).

\section{CONCLUSÕES}

A predominância de picos em $n$ C29 e $n$ C31 associada à predominância de $n$-alcanos ímpares sobre os pares para o intervalo entre $n \mathrm{C} 23$ e $n \mathrm{C} 31$ fornece um forte indício de origem biogênica continental a partir de vegetais superiores para a matéria orgânica estocada nos sedimentos. Os picos em $n \mathrm{C} 25$ podem estar relacionados com bactérias não fotossintéticas, muito comuns em manguezais, enquanto que o pico em $n \mathrm{C} 23$ pode estar relacionado com a presença de óleo lubrificante no ambiente (Aboul-Kassim \& Simoneit, 1995). A ausência de picos na faixa dos $n$-alcanos leves não pode ser interpretada como ausência de contribuição de matéria orgânica marinha, uma vez que os compostos mais leves são facilmente degradados em amostras ambientais sob as condições de clima tropical (Woodhead et al., 1999). Em ambientes complexos como os manguezais de influência marinha e continental, espera-se que coexistam compostos orgânicos de origens diversas, relacionadas aos diferentes agentes reguladores locais. Acrescenta -se o fato que, na região norte da Baía de Todos os Santos, ainda se deve considerar o aporte antropogênico relacionado à indústria petrolífera e aos lançamentos de esgotos industriais e domésticos (Woodhead et al., 1999; Lê Dréau et al., 1997).

Os padrões descritos indicam uma origem complexa para a matéria orgânica estocada no sedimento, relacionada à vegetais superiores, provavelmente ligada ao manguezal, derrames acidentais de óleos diversos e /ou lançamentos de esgotos domésticos e industriais, além de um alto grau de degradação dos óleos derramados no ambiente.

Os biomarcadores saturados cíclicos identificados nos sedimentos comprovam a presença de petróleo e derivados nos ecossistemas de manguezal, caracterizando um cenário de poluição por compostos orgânicos relacionados ao complexo petrolífero. Quanto às características dos óleos, esses biomarcadores apontam para uma mistura de óleos de origens distintas e indicam a presença de óleo correlacionável àquele produzido na Bacia do Recôncavo.

\section{AGRADECIMENTOS}

Este trabalho é um reflexo do apoio financeiro concedido pela FINEP - CTPETRO - FAPEX PETROBRAS - CNPq (no. 2201074500) Agência Nacional de Petróleo (ANP) e pelo financiamento das análises laboratoriais através Fundação Universidade Norte Fluminense (FENORTE).

\section{REFERÊNCIAS}

Aboul-Kassim, T.A.T.; Simoneit, B.R.T. 1995. Petroleum hydrocarbon fingerprinting and sediment transport assessed by molecular biomarker and multivariate statistical analyses in the eastern Harbour of Alexandria, Egypt. Marine Pollution Bulletin, 30 (1): $63-73$. 
Celino, J.J.; Queiroz, A.F.S; Santos, J.B. 2005. Heavy metals and petroleum hydrocarbons in sediments of the mangrove from São Francisco do Conde Region - Todos os Santos Bay - Bahia. $3^{\circ}$ Congresso Brasileiro de P\&D em Petróleo e Gás, Salvador, Bahia, 220-230.

Celino, J.J. \& Queiroz, A.F.S. 2006. Fonte e grau da contaminação por hidrocarbonetos policíclicos aromáticos (HPAs) de baixa massa molecular em sedimentos da Baía de Todos os Santos, Bahia. Revista da Escola de Minas, 59(3): 265-270.

Le Dréau, Y.; Jacquot, F.; Doumenq, P.; Guiliano, M.; Bertrand, J.C.; Mille, G. 1997. Hydrocarbon balance of a site which had been highly and chronically contaminated by petroleum wastes of a refinery (from 1956 to 1992). Marine Pollution Bulletin, 34 (6): $456-468$.

Leão, Z.M.A.N., Dominguez, J.M.L. 2000. Tropical coast of Brazil. Marine Pollution Bulletin. (41):112-122.

Orge, M.D.R.; Porsché, I.J.; Costa, M.C.; Lima, J.S.; Soares, S.E.D.; Justino, R. 2000. Assessment of oil refinery waste on Rhizophora mangle $L$. seedling growth in mangrove of Todos os Santos Bay, Bahia, Brazil - Aquatic Ecosystem Health and Management. (3): 471-477.

Peso -Aguiar, M.C., Smith, D.H., Assis, R.C.F., Santa - Isabel, L.M., Peixinho, S., Gouveia, E.P., Almeida,
T.C.A., Andrade, W.S., Carqueija, C.R.G., Kelmo, F., Carrozzo, G., Rodrigues, C.V., Carvalho, G.C., Jesus, A.C.S. 2000. Effects of petroleum and its derivatives in benthic communities at Baía de Todos os Santos, Bahia, Brazil. Aquatic Ecosystem Health and Management. (3): 459-470.

Peters, K. E.; Moldowan, J. M. 1993. The biomarker guide: interpreting molecular fossils in petroleum and ancient s sediments. Prentice Hall, Englewood Cliffs, New Jersey, 362p.

Tavares, T.M. 1997. Contaminação química no ambiente marinho in Baía de Todos os Santos: diagnóstico sócio - ambiental e subsídios para a gestão Germen / Universidade Federal da Bahia - NIMA Salvador, $151-164$.

Veiga, I.G. 2003. Avaliação da origem dos hidrocarbonetos em sedimentos superficiais de manguezais da região norte da Baía de Todos os Santos, Bahia. Dissertação de mestrado. Laboratório de Engenharia e Exploração de Petróleo - LENEP/UENF. 205p.

Woodhead, R.J.; Law, R.J.; Matthiessen, P. 1999. Polycyclic aromatic hydrocarbons in surface sediments around England and Wales, and their possible biological significance. Marine Pollution Bulletin, 38 (9): $773-790$. 